Abstract IDDF2021-ABS-0146 Table 6 Binary Logistic Regression for the Association of the Indications for Colonoscopy with the Selected Colonoscopy Findings among the Respondents ( $N=196)$

\begin{tabular}{|c|c|c|c|c|c|c|}
\hline \multicolumn{7}{|c|}{ Colonoscopy Findings } \\
\hline & \multicolumn{2}{|c|}{ Normal Colon } & \multicolumn{2}{|c|}{ Non-Specific Colitis } & \multicolumn{2}{|c|}{ Colonic Polyp } \\
\hline & $\mathrm{OR}$ & $p$-value & $\mathrm{OR}$ & $p$-value & $\mathrm{OR}$ & $p$-value \\
\hline Hematochezia & $-4.47^{\dagger}$ & 0.002 & 1.42 & 0.323 & $9.23^{\dagger}$ & 0.003 \\
\hline Constipation & 2.56 & 0.413 & -1.48 & 0.669 & 1.00 & - \\
\hline Melena & $-4.86^{*}$ & 0.032 & $-9.62^{*}$ & 0.034 & -1.51 & 0.609 \\
\hline Diarrhea & 1.10 & 0.927 & 4.36 & 0.067 & 1.00 & - \\
\hline Abdominal Pain & 3.76 & 0.066 & -1.40 & 0.577 & 1.00 & - \\
\hline Weight Loss & 1.00 & - & 1.00 & - & 1.00 & - \\
\hline Occult Bleeding & 1.00 & - & 1.00 & - & 1.00 & - \\
\hline \multirow[t]{4}{*}{ Surveillance } & 1.00 & - & 1.00 & - & 1.00 & - \\
\hline & \multicolumn{2}{|c|}{ Inflammatory } & \multicolumn{2}{|l|}{ Crohn's } & \multicolumn{2}{|c|}{ Ulcerative Colitis } \\
\hline & \multicolumn{2}{|c|}{ Bowel Disease } & \multicolumn{2}{|l|}{ Disease } & & \\
\hline & $\mathrm{OR}$ & $p$-value & $\mathrm{OR}$ & $p$-value & $\mathrm{OR}$ & $p$-value \\
\hline Hematochezia & 1.29 & 0.818 & -3.97 & 0.333 & -1.31 & 0.818 \\
\hline Constipation & 1.00 & - & 1.00 & - & $15.67^{*}$ & 0.029 \\
\hline Melena & 1.00 & - & 1.00 & - & 1.00 & - \\
\hline Diarrhea & 1.00 & - & 1.00 & - & 1.00 & - \\
\hline Abdominal Pain & 3.25 & 0.300 & 1.00 & - & 1.00 & - \\
\hline Weight Loss & 1.00 & - & 1.00 & - & 1.00 & - \\
\hline Occult Bleeding & 1.00 & - & 1.00 & - & 1.00 & - \\
\hline Surveilliance & 1.00 & - & 1.00 & - & 1.00 & - \\
\hline \multicolumn{7}{|l|}{${ }^{*}$ Significant at 0.05} \\
\hline${ }^{\dagger}$ Significant at 0.01 & & & & & & \\
\hline
\end{tabular}

considered in a young child presenting with hematochezia. Chronic constipation should warrant investigation for IBD as the incidence of this disease is rising. A prospective multi-center study is recommended to identify the true burden of pediatric colonic diseases, especially in IBD.

\section{IDDF2021-ABS-0147 FECAL CALPROTECTIN IS SPECIFIC IN PREDICTING ORGANIC BOWEL DISEASES}

Liqiaona Fan*, Muzhou Han, Siying Zhu, Shutian Zhang, Haiyun Shi. Department of Gastroenterology, Beijing Friendship Hospital, Capital Medical University, China

\subsection{6/gutjnl-2021-IDDF.158}

Background Although colonoscopy remains the gold standard for determining bowel diseases, it's an invasive test, which is not suitable for the initial screening for organic bowel diseases. We aimed to investigate the value of fecal calprotectin (FC) for the differentiation of organic bowel diseases and functional bowel disorders.

Methods In this prospective study, patients were included if they underwent colonoscopy for any symptom of the lower digestive tract or colorectal cancer (CRC) screening. Stool samples for FC measurement were collected within 3 days before the colonoscopy. Predictive parameters of FC in discriminating organic bowel diseases (CRC, advanced adenoma and bowel inflammation) were evaluated.

Results Of 192 patients included, 51\% were men, median age was 57 (interquartile range, 42-65) years old. 103 patients had organic bowel diseases (CRC, $n=37$; advanced adenoma, $\mathrm{n}=32$; bowel inflammation, $\mathrm{n}=34$ ). $\mathrm{FC}$ had an area under the curve of $0.750 \quad(95 \%$ confidence interval $0.683-0.810, \mathrm{p}<0.001)$ for determining organic bowel diseases. FC level $>100 \mathrm{ug} / \mathrm{g}$ predicted organic bowel diseases with a sensitivity, specificity, positive predictive value and negative predictive value of $62.14 \%, 80.90 \%, 79.01 \%$ and $64.86 \%$, respectively.

Conclusions FC has high specificity in predicting organic bowel diseases. Colonoscopy should be performed in patients with a high level of FC.

\section{IDDF2021-ABS-0148 GUIDED RELEASE OF A BREATH (GRAB) - BRINGING UREA BREATH TEST (UBT) TO THE DOORSTEP}

${ }^{1}$ Low Yue Wey ${ }^{*}$, ${ }^{2}$ Siah Tien Ho Kewin, ${ }^{2}$ Margaret Teng, ${ }^{2}$ Phyllis Chin, ${ }^{2}$ Dai Jiawen, ${ }^{2}$ Yu Sen Alex Soh, ${ }^{2}$ Jonathan Lee. ${ }^{1}$ Yong Loo Lin School of Medicine, Singapore; ${ }^{2}$ Division of Gastroenterology and Hepatology, Department of Medicine, National University Hospital, Singapore

\subsection{6/gutjpl-2021-IDDF.159}

Background Urea Breath Tests (UBTs) are common non-invasive tests performed in hospitals under the guidance of trained technicians. However, COVID-19 has reduced access to within-hospital UBTs. Self-conducted UBT (SUBT) at home has been suggested as an alternative. This study aims to compare the efficacy of SUBT with written compared to video instructions.

Methods The study was divided into 3 parts. In Part 1, consecutive first-time UBT patients were randomized to receive either written or video instructions. In Part 2, the original video was enhanced. For Parts 1 and 2, a competency score of 1-5 based on the assessment of the 5 key steps was determined by a trained observer. Having reviewed the steps for breath collection in the first 2 parts, we went on to Part 3 to determine if breath collection with these steps would be successful. A further enhanced instructional video was created and successful collection of breath was recorded based on detection of sufficient recovered C-13 in the breath bag for the baseline test.

Results In total, 129 patients were recruited for the study. Patients excluded for this study included those who had visual or hearing impairment, cognitive impairment and those who did not understand English as our instructional materials were in English. For Part 1, 20 patients were randomised into written $(n=10)$ and original video $(n=10)$ groups. There was numerical improvement but no statistically significant difference in median scores for written and video instructions (2 vs 4, $\mathrm{p}=0.055)$. A further 10 patients were recruited for Part 2, the enhanced video group. Median scores were statistically improved for enhanced video compared to written (5 vs 2, $\mathrm{p}=0.043$ ). In Part 3, 99 patients were recruited. Barring machine fault, all the UBTs were successful without repeating the breath test.

Conclusions An enhanced instructional video is highly successful at enabling SUBT. This can improve the safety of patients and operators by allowing UBTs to be conducted at home, while maintaining accuracy. This would reduce the time that patients spend in the hospital and reduce exposure to potential COVID infections. 